\title{
Role of Computed Tomography in the Evaluation of Pediatric Retroperitoneal Masses: A Retrospective Study
}

\section{Gupta Vikrant $^{1}$, Abrol Deepak ${ }^{2}$}

${ }^{1}$ Department of Radio-Diagnosis and Imaging, Government Medical College, Jammu, India. ${ }^{2}$ Department of Radiation Oncology, Government Medical College, Kathua, India.

\begin{abstract}
Objective: Retroperitoneum is one of the largest spaces in the body. Retroperitoneal (RP) masses are common in paediatric age group and present with vague clinical features. Computed Tomography (CT) is an excellent imaging modality in the evaluation of RP masses. The aim of this study was to evaluate the role of CT in the detection and characterization of RP masses. Materials and Methods: 28 clinically suspected or sonographically detected RP masses formed the material of the study. Detailed clinical history was elicited from the patients/ attendants and findings of general physical examination recorded. Non-contrast and Contrast Enhanced CT was performed and masses were evaluated with respect to site of origin, consistency, components and pattern of enhancement. Probable CT diagnosis was made and findings correlated with pathological/laboratory findings wherever possible. The results were subjected to statistical analysis and expressed as percentages and proportions. Results: Majority of patients were seen in 1-5 years age group with M:F ratio of 1.8:1. Lump abdomen was the commonest presenting feature followed by pain abdomen. Lymph nodal masses were the commonest primary RP space mass while renal masses accounted for the majority of secondary RP masses in our study. Pathological/ Laboratory correlation was available in 21 cases and CT was able to differentiate benign and malignant lesions in $95.2 \%$ cases. Overall diagnostic accuracy of $\mathrm{CT}$ in the evaluation of RP masses was $85.7 \%$. Conclusions: $\mathrm{CT}$ is a simple and reliable tool in diagnosing pediatric RP masses with a high diagnostic accuracy.
\end{abstract}

Keywords: Retroperitoneum- Malignant- Wilm's Tumour- Computed Tomography

Asian Pac J Cancer Care, 6 (3), 237-241

\section{Introduction}

The Retroperitoneal (RP) space is bounded anteriorly by the peritoneal covering, posteriorly by posterior abdominal wall, superiorly by the $12^{\text {th }}$ rib and vertebra, inferiorly by the iliac crest and laterally by the quadratus lumborum muscle. The contents of retroperitoneum include connective tissue, adrenals, kidneys, ureters, aorta and its branches, inferior vena cava and its tributaries and lymph nodes.

RP masses pose a diagnostic challenge for the treating clinician. Diagnostic evaluation, including radiology and pathology play a paramount role in the evaluation of RP masses. These may be congenital or acquired, benign or malignant, arising primarily from RP space, directly from the major RP organs or secondarily involving the RP
Submission Date: 10/05/2020Ａcceptance Date: 01/26/2021

\footnotetext{
Corresponding Author:

Dr. Vikrant Gupta

Department of Radio-Diagnosis and Imaging, Government Medical College, Jammu, India.

Email: vikrantgupta884@gmail.com
} 
is operator dependent and limited by artefacts from bone and air. Magnetic Resonance Imaging (MRI) has a unique role in diagnosis of RP masses. MRI offers advantages of excellent soft tissue contrast with high spatial resolution and lack of ionizing radiation. However MRI is a lengthy investigation, not readily available and is costly [2]. Also MRI in children requires sedation and is susceptible to motion artefacts.

Computed Tomography (CT) is an excellent cross sectional imaging technique in the evaluation of RP masses. It provides explicit anatomical details, faster data acquisition times, higher spatial resolution, large volume coverage and reduced image artefacts. CT scan can precisely evaluate anatomical and morphological characters of the mass, site of origin, extent and metastatic spread. CT is the most powerful and versatile procedure in evaluation of RP masses in children [3]. The advent of Multi-Detector Computed Tomography (MDCT) has resulted in improved resolution and considerable reductions in scan acquisition and display times [4]. So the current study was performed to evaluate the role of $\mathrm{CT}$ the in detection and characterization of RP masses.

\section{Materials and Methods}

This retrospective observational study was carried out in North-West India over a period of one year. All patients with clinically suspected or USG detected RP masses referred for CT evaluation formed the material of the study. Patients with allergy to iodinated contrast media, patients with renal failure or on dialysis and patients with acute abdominal trauma were excluded from the study. Detailed clinical history was elicited from the patients/ attendants and findings of general physical examination recorded. Renal Function Tests were performed in all the patients and the study performed with minimum 6 hours fasting. CT examination was performed using MDCT machines (GE Bright speed 16 slice and Siemens Somatom Definition AS+ 64 slice). Non contrast CT was first performed followed by contrast enhanced CT (portal venous phase) using non-ionic low osmolar contrast media (concentration between $300-400 \mathrm{mg} / \mathrm{ml}$ and dose of $2 \mathrm{ml} /$ $\mathrm{kg}$ body weight). Oral and Rectal contrast media was used wherever indicated. Angiographic and delayed sequences were obtained wherever deemed necessary. Multiplanar and 3-D reconstructions were performed on dedicated work station. The lesions were evaluated with respect to site of origin, consistency, components of the lesion and pattern of enhancement. Imaging findings were correlated with the pathological/laboratory findings wherever possible. The results were subjected to statistical analysis and expressed as percentages and proportions.

\section{Results}

28 clinically suspected or USG documented patients with RP masses formed the material of the study. Age of the patients ranged from 1 year to 17 years. Majority of patients were seen in 1-5 years age group followed by 11-15 years age group. Out of 28 patients, 18 were male and 10 were female with M:F ratio of 1.8:1. The commonest presenting features were lump abdomen (71.4\%) followed by pain abdomen $(53.6 \%)$.

On MDCT, the lesions were subdivided into Primary RP space masses and lesions originating from RP organs (Table 1). Lymph nodal masses were the commonest primary RP space masses (14.3\%) in our study. Two cases each of teratoma and lymphangioma were seen while solitary case each of Paraganglioma and Duplication Cyst were seen. Two primary RP space masses appeared as heterogeneously enhancing mass on MDCT and could not be definitely diagnosed (Table 1). These were suspected

Table 1. Distribution of Pediatric Retroperitoneal Masses on MDCT ( $\mathrm{n}=28)$

\begin{tabular}{llcc}
\hline MDCT & Number & Percentage (\%) \\
\hline Primary RP space masses & 1. Lymph Nodal Masses & & 10.7 \\
& A) Lymphoma & 3 & 3.6 \\
& B) Infective & 1 & 7.1 \\
& 2. Teratoma & 2 & 7.1 \\
3. Lymphangioma & 2 & 3.6 \\
& 4. Paraganglioma & 1 & 3.6 \\
5. Duplication Cyst & 1 & 7.1 \\
6. Non-Diagnostic & 2 & 14.3 \\
& 1. Wilm's Tumour & 4 & 7.1 \\
& 2. Abscess & 2 & 3.6 \\
3. Angiomyolipoma & 1 & 3.6 \\
4. Multicystic Dysplastic Kidney & 1 & 3.6 \\
5. Multilocular Cystic nephroma & 1 & 3.6 \\
& 6. Renal Lymphoma & 1 & 10.7
\end{tabular}


Table 2. Radiological and Pathological/Laboratory correlation $(n=21)$

\begin{tabular}{|c|c|c|c|c|}
\hline \multirow[t]{2}{*}{ Diagnostic Categories } & \multirow[t]{2}{*}{ MDCT Diagnosis } & \multicolumn{2}{|c|}{ Pathological/Laboratory Diagnosis } & \multirow{2}{*}{$\begin{array}{c}\text { Diagnostic Accuracy } \\
(\%)\end{array}$} \\
\hline & & Concordant & Discordant & \\
\hline \multirow[t]{6}{*}{ Primary RP space masses } & 1. Nodal Masses & 3 & 1 & 66.70 \\
\hline & 2. Teratoma & 1 & - & 100 \\
\hline & 3. Lymphangioma & 1 & - & 100 \\
\hline & 4. Paraganglioma & - & - & 0 \\
\hline & 5. Duplication Cyst & 1 & - & 100 \\
\hline & 6. Non-Diagnostic & - & 2 & 0 \\
\hline \multirow[t]{6}{*}{ Renal } & 1. Wilm's Tumour & 3 & - & 100 \\
\hline & 2. Abscess & 1 & - & 100 \\
\hline & 3. Angiomyolipoma & 1 & - & 100 \\
\hline & 4. Multicystic Dysplastic Kidney & 1 & - & 100 \\
\hline & 5. Multilocular Cystic nephroma & - & - & - \\
\hline & 6. Renal Lymphoma & 1 & - & 100 \\
\hline \multirow[t]{3}{*}{ Adrenal } & 1. Neuroblastoma & 2 & - & 100 \\
\hline & 2. Pheochromocytoma & 2 & - & 100 \\
\hline & 3. Adrenomyelipoma & 1 & - & 100 \\
\hline Total & & 18 & 3 & 85.70 \\
\hline
\end{tabular}

to be malignant in nature based on CT findings.

Renal masses accounted for majority of secondary RP masses in our study (35.7\%). These included 4 cases of Wilms Tumour, 2 cases of renal abscess and solitary case each of renal angiomyolipoma, multicystic dysplastic kidney, multilocular cystic nephroma and renal lymphoma (Table 1). The adrenal masses observed in our study included neuroblastoma (3 cases), pheochromocytoma ( 2 cases) and adrenomyelolipoma (1 case) (Table 1$)$.

Pathological/Laboratory correlation was available in 21 cases only as rest of the cases were lost to follow up. MDCT was able to differentiate benign and malignant lesions in $95.2 \%$ cases as one case of nodal lymphoma on MDCT proved as granulomatous pathology on fine needle

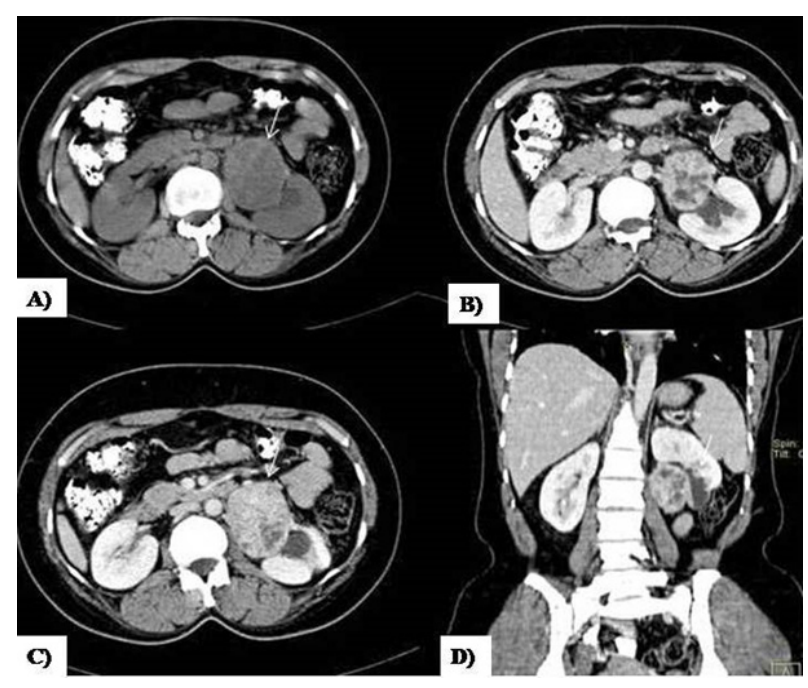

Figure 1. Paraganglioma, Non contrast (A) and Contrast Enhanced (B,C,D) CT images reveal a large ill defined heterogeneously enhancing mass with areas of necrosis abutting and displacing left kidney. aspiration. Also the two undiagnosed heterogeneously enhancing primary RP space masses on MDCT were diagnosed as Primitive Neuroectodermal Tumour (PNET) and Extra-Gonadal Germ Cell (EGGCT) Tumour on histopathology. So overall diagnostic accuracy of MDCT in evaluation of RP masses was $85.7 \%$ (Table 2 ).

\section{Discussion}

Pediatric RP masses include a varied spectrum of lesions with diverse origin and significance. These may occur at any age from the neonatal period through adolescence and patient's age is one of the important factors that narrow the potential differentials. In majority of the cases, RP masses are detected late and present with nonspecific symptoms. Large proportion of the primary RP masses are malignant and so radiological and pathological evaluation is necessary to identify the precise anatomic location and extent of the RP masses and to differentiate benign from malignant lesions.

28 patients with RP masses formed the material of the study. The age range of the patients varied from 1 year to 17 years. Majority of patients were seen in 0-5 years age group followed by 11-15 years age group with a $\mathrm{M}: \mathrm{F}$ ratio of 1.8:1. Nagaraju RM et al[5] observed majority of abdominal masses in age range of 0-5 years. Abdominal lump was the commonest clinical complaint (71.4\%) followed by pain abdomen $(53.6 \%)$.

On the basis of MDCT findings, the lesions were characterised as benign or malignant and were categorised into Primary RP space masses and lesions originating from RP organs (Table 1).

Enlarged Lymph nodes were the commonest primary RP space lesions seen in 4 (14.7\%) cases. In 3 of 4 patients, MDCT showed well defined homogenous and discrete 


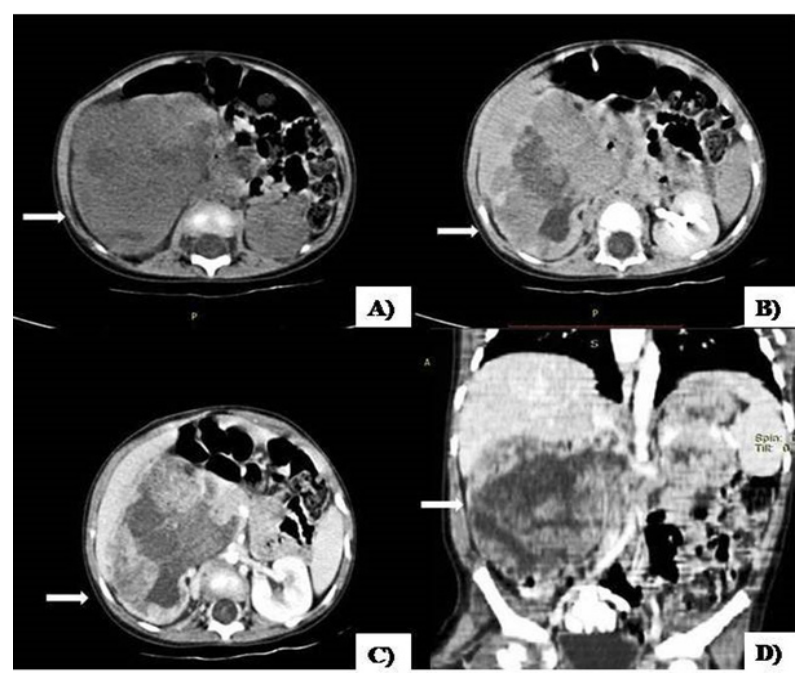

Figure 2. Wilm's Tumor, Non contrast (A) and Contrast Enhanced (B,C,D) CT images reveal a large ill defined heterogeneously enhancing lesion with necrosis almost replacing entire right kidney, The lesion is seen crossing midline with displacement of adjacent viscera and vessels

enlarged RP lymph nodes. No evidence of any necrosis or calcification was seen in these cases. In all these cases, evidence of similar enhancing enlarged lymph nodes were seen elsewhere in the body and based on these findings, a provisional MDCT diagnosis of lymphoma was made. Pathological correlation was performed in all the patients. Correct MDCT diagnosis was made in 2 patients while the $3^{\text {rd }}$ patient was diagnosed as tuberculosis as granulomas were seen on cytology. In the $4^{\text {th }}$ patient, heterogeneously enhancing lymph nodal mass with internal necrosis and evidence of conglomeration was observed on MDCT with probable diagnosis of tuberculosis which was confirmed pathologically.

Two cases of teratoma were seen on MDCT. MDCT in both the cases revealed large well defined RP mass with presence of cystic and fat attenuation components and displacement of adjacent bowel loops. Bony structures/calcifications were also seen in the mass. Teratoma is the third commonest RP tumour in the pediatric age group after neuroblastoma and Wilms' tumour and constitute $1-11 \%$ of primary RP tumours [6]. Two cases of Lymphangiomas were also observed in our study. Lymphangiomas are benign vascular lesions showing lymphatic differentiation. Majority (95\%) of lymphangiomas occur in the neck and axilla while remaining $5 \%$ are seen in the mesentery, retroperitoneum, abdominal viscera, lung and mediastinum [7]. Contrast enhanced CT reveals ill defined fluid density lesion and may show enhancement of the cyst wall and septa. The fluid component is typically homogeneous with low attenuation values and occasionally negative attenuation values may occur in the presence of chyle [7]. Single case of Teratoma and lymphangioma were subjected to surgical and pathological evaluation and results correlated with MDCT findings.

Solitary case each of Paraganglioma and Duplication

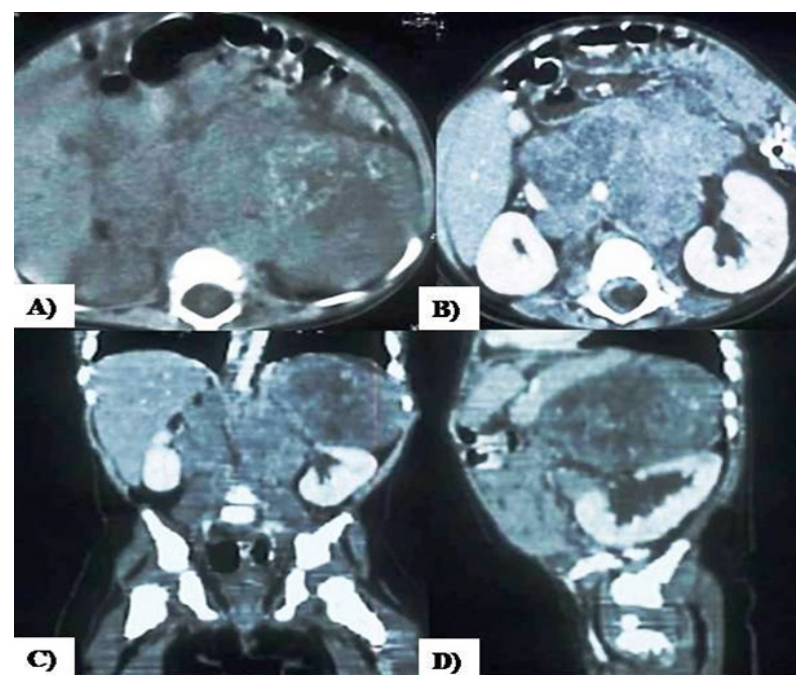

Figure 3. Neuroblastoma, Non contrast (A) and Contrast Enhanced (B,C,D) CT images reveal a large ill defined heterogeneously enhancing mass with necrosis and calcifications arising from left suprarenal fossa, crossing midline and encasing aorta and displacing adjacent structures.

Cyst were also seen. Paragangliomas or extra-adrenal pheochromocytomas are rare neurogenic tumors arising from highly vascularised neural crest cells called paraganglia that are symmetrically distributed along the aortic axis. According Hayes WS et al [8], 10\% to $20 \%$ of pheochromocytomas are extra-adrenal and often arise in the retroperitoneum from the organs of Zuckerkandl. On MDCT, paragangliomas appear as well defined homogenous or heterogeneously enhancing lesions with intense intense contrast enhancement. No evidence of fat, hemorrhage or calcification was observed (Figure 1).

Two primary RP space masses appeared as heterogeneously enhancing masses on MDCT and could not be definitely diagnosed. Both the lesions underwent surgical and histopathological evaluation and were diagnosed as PNET and EGGCT.

Four cases of Wilm's tumour were seen in our study and all were less than 5 years of age. Geller E et al [9] observed peak incidence of Wilm's tumor in 3-4 years of age while Kaste SC et al [10] observed the peak incidence in $<2$ years of age. The lesions appeared as heterogeneously enhancing lesions involving the kidney with ill-defined borders and displacing adjacent structures (Figure 2). Fat, calcification and hemorrhage were not seen while renal vein involvement was seen in one case. Histopathological correlation was available in 3 cases and $100 \%$ correlation was observed between MDCT and pathological diagnosis.

Three cases of neuroblastoma were seen in our study and all cases were seen under 2 years of age. On MDCT, neuroblastoma appears hypodense on non-contrast CT with heterogenous post contrast. The lesions crossed midline and encased the surrounding vessels. The foci of calcifications and necrosis were evident in the lesion (Figure 3) similar to the findings of Lowe LH et al. [11] Woodward PF et al [12] found that calcification, a common 
feature in paediatric neuroblastoma. Histopathological correlation was available in 2 cases and $100 \%$ correlation was observed between MDCT and pathological diagnosis.

Two cases of adrenal gland pheochromocytoma on MDCT were observed in our study. History of hypertension was present in both cases. Pheochromocytomas can be isolated or associated with multiple endocrine neoplasia (MEN) types IIA and IIB, von Hippel-Lindau disease, neurofibromatosis, Sturge-Weber syndrome, Carney's triad, and isolated familial pheochromocytoma. On MDCT, Pheochromocytoma usually show intense contrast enhancement. Heterogenous enhancement and calcification may also be seen in few cases. No evidence of haemorrhage, fat content, vessel involvement and intraspinal and midline extension was noted in any of the cases in our study. Urinary catecholamine levels were found to be elevated in both the cases confirming the CT diagnosis.

Pathological/Biochemical correlation was available in 21 cases and rest of the cases were lost to follow up. One case of nodal lymphoma on MDCT proved as granulomatous pathology on FNAC. Also the two heterogeneously enhancing primary RP space masses on MDCT were diagnosed as Primitive Neuroectodermal Tumour (PNET) and Extra-Gonadal Germ Cell (EGGCT) Tumour on histopathology. So MDCT was able to differentiate benign and malignant lesions in $95.2 \%$ cases and overall diagnostic accuracy of MDCT in evaluation of RP masses was $85.7 \%$.

There were a few limitations in our study. Firstly the sample size was small and so more such studies should be carried out in future to further substantiate the results obtained in our study. Another limitation of the study was lack of MRI correlation with MDCT findings as MRI has better soft tissue resolution and better sensitivity in evaluation of RP masses. However performing MRI is difficult in pediatric age group as children are mostly uncooperative and MRI examination takes significantly longer time as compared to MDCT.

Hence we conclude that knowledge of RP anatomy on $\mathrm{CT}$ enables adequate differentiation between primary and secondary RP space masses. CT is a simple, minimally invasive and reliable tool in diagnosing pediatric RP masses with a high diagnostic accuracy. Also CT can differentiate benign and malignant masses in nearly all the cases and thus can serve as a handy tool in deciding appropriate management and outcome in these patients.

\section{Acknowledgements}

\section{Nil}

\section{Funding}

This research did not receive any specific grant from funding agencies in the public, commercial, or not-forprofit sectors.

\section{Conflict of interest}

"The authors declare no conflict of interest."

\section{References}

1. Caty MG, Shamberger RC. Abdominal Tumors in Infancy and Childhood. Pediatric Clinics of North America. 1993 Dec;40(6):1253-1271. https://doi.org/10.1016/s00313955(16)38660-6

2. Hugosson C, Nyman R, Jorulf H, McDonald P, Rifai A, Kofide A, Jacobsson B. Imaging of Abdominal Neuroblastoma in Children. Acta Radiologica. 1999 09;40(5):534-542. https:// doi.org/10.3109/02841859909175580

3. Shaw A, Dixon A. Multidetector Computed Tomography. In: Adam A, Dixon AK, editors. Grainger \& Allison's Diagnostic Radiology: A textbook of Medical Imaging. 5th ed. London: Elsevier Ltd. 2008;:79-86.

4. Gupta S, Razak K, Meena G. Exploring the Pediatric Abdomen: A Radiological Evaluation. J Bio Innov. 2018 Jan;7(1):47-65.

5. Nagaraju R, Bhimarao. Role of Multidetector Computed Tomography in Evaluation of Pediatric Abdominal Tumors. J Evol Med Dent Sci. 2015 May;4(42):7352-64.

6. Nguyen CT, Kratovil T, Edwards MJ. Retroperitoneal teratoma presenting as an abscess in childhood. Journal of Pediatric Surgery. 2007 Nov;42(11):e21-e23. https://doi. org/10.1016/j.jpedsurg.2007.07.053

7. Lugo-Olivieri CH, Taylor GA. CT differentiation of large abdominal lymphangioma from ascites. Pediatric Radiology. 1993 04;23(2):129-130. https://doi.org/10.1007/bf02012405

8. Hayes WS, Davidson AJ, Grimley PM, Hartman DS. Extraadrenal retroperitoneal paraganglioma: clinical, pathologic, and CT findings.. American Journal of Roentgenology. 1990 Dec;155(6):1247-1250. https://doi. org/10.2214/ajr.155.6.2173385

9. Geller E, Kochan PS. Renal Neoplasms of Childhood. Radiologic Clinics of North America. 2011 07;49(4):689709. https://doi.org/10.1016/j.rcl.2011.05.003

10. Kaste SC, Dome JS, Babyn PS, Graf NM, Grundy P, Godzinski J, Levitt GA, Jenkinson H. Wilms tumour: prognostic factors, staging, therapy and late effects. Pediatric Radiology. 2007 Nov 17;38(1):2-17. https://doi.org/10.1007/ s00247-007-0687-7

11. Lowe LH, Isuani BH, Heller RM, Stein SM, Johnson JE, Navarro OM, Hernanz-Schulman M. Pediatric Renal Masses: Wilms Tumor and Beyond. RadioGraphics. 2000 Nov;20(6):1585-1603. https://doi.org/10.1148/ radiographics.20.6.g00nv051585

12. Woodward PJ, Sohaey R, Kennedy A, Koeller KK. From the Archives of the AFIP. RadioGraphics. 2005 01;25(1):215242. https://doi.org/10.1148/rg.251045156

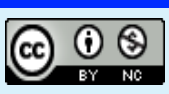

This work is licensed under a Creative Commons AttributionNon Commercial 4.0 International License. 